\title{
Imaging Nonradiative Point Defects Buried in Quantum Wells Using Cathodoluminescence
}

Thomas F. K. Weatherley, * Wei Liu, Vitaly Osokin, Duncan T. L. Alexander, Robert A. Taylor, Jean-François Carlin, Raphaël Butté, and Nicolas Grandjean

Cite This: Nano Lett. 2021, 21, 5217-5224

Read Online

ACCESS | Llll Metrics \& More | 国 Article Recommendations ｜９） Supporting Information

ABSTRACT: Crystallographic point defects (PDs) can dramatically decrease the efficiency of optoelectronic semiconductor devices, many of which are based on quantum well (QW) heterostructures. However, spatially resolving individual nonradiative PDs buried in such QWs has so far not been demonstrated. Here, using high-resolution cathodoluminescence (CL) and a specific sample design, we spatially resolve, image, and analyze nonradiative PDs in InGaN/GaN QWs at the nanoscale. We identify two different types of PDs by their contrasting behavior with temperature and measure their densities from $10^{14} \mathrm{~cm}^{-3}$ to as high as $10^{16} \mathrm{~cm}^{-3}$. Our CL images clearly illustrate the interplay between PDs and carrier dynamics in the well: increasing PD concentration severely limits carrier diffusion lengths, while a higher carrier density suppresses the nonradiative behavior of PDs. The results

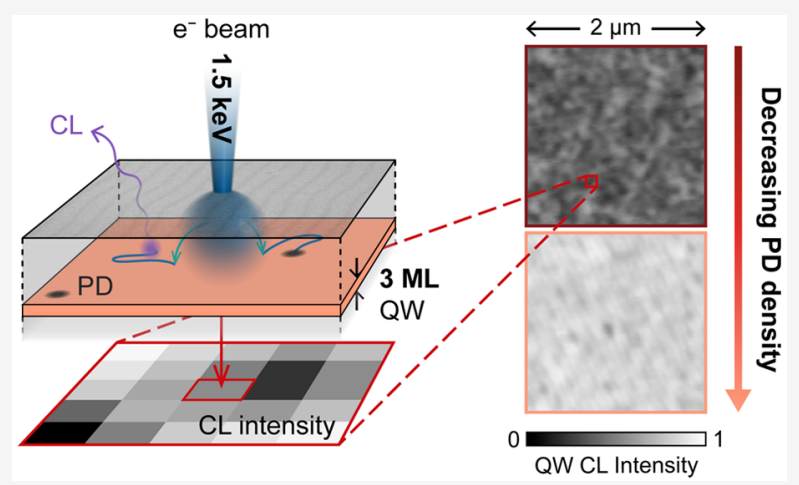
in this study are readily interpreted directly from CL images and represent a significant advancement in nanoscale PD analysis.

KEYWORDS: semiconductor, point defects, quantum wells, cathodoluminescence, InGaN, imaging

$\mathrm{C}$ rystallographic point defects (PDs) have a profound effect on the optical properties of semiconductors. ${ }^{1}$ Of particular interest are PDs with energy levels deep in the bandgap, which can act as efficient electron-hole recombination centers. ${ }^{2}$ Recombination at PDs can be useful if it leads to stable single-photon emission, as is the case, for example, for silicon/nitrogen-vacancy centers in diamond. ${ }^{3,4}$ However, it is more common for PD recombination to proceed via multiplephonon emission resulting in no light emission at all, ${ }^{5}$ and such nonradiative PDs can dramatically decrease the internal quantum efficiency (IQE) of optoelectronic devices such as light-emitting diodes (LEDs), laser diodes, and solar cells. ${ }^{6-8}$ Despite the relevance of these defects to both industry and research, spatially resolving, imaging, and analyzing individual PDs on the nanoscale remains a serious challenge, particularly at high defect densities. Previous studies have analyzed surface defects or PDs in $2 \mathrm{D}$ materials ${ }^{9-14}$ but have not imaged PDs buried in semiconductor heterostructures due to the difficulty of pinpointing atomic-scale defects in bulk. Consequently, PDs have not been individually imaged in quantum wells (QWs), even though such wells are the active region in the majority of today's LEDs, as well as the core of emerging nanostructures such as nanobeam lasers. ${ }^{15}$ Imaging the nonradiative PDs that plague these devices is particularly challenging since they lack any localized luminescence, ruling out the use of superresolution techniques. ${ }^{12,16}$

The critical role of nonradiative PDs in QWs is exemplified perfectly by III-nitride semiconductors: recent literature indicates that an intrinsic PD in InGaN/GaN QWs acts as a highly effective nonradiative recombination center, killing the IQE of green to near-ultraviolet LEDs. ${ }^{17-21}$ These PDs arise during growth from an initial population of GaN surface defects which are only incorporated into layers with indium content. $^{21,22}$ Growing an indium-containing "underlayer" below the QW can therefore trap the surface defects before they incorporate into the well. Varying the thickness of this underlayer allows for precise PD density control in the QW, making III-nitrides the ideal platform to explore nonradiative PD imaging.

Here, using high-resolution cathodoluminescence $(\mathrm{CL})$ we spatially resolve and analyze individual nonradiative PDs in ultrathin InGaN/GaN QWs up to densities as high as $10^{16}$ $\mathrm{cm}^{-3}$. We identify two different types of PDs in the QWs and show that one type, linked to a midgap deep state, decreases the macroscale room-temperature IQE from around $64 \%$ to only $1 \%$ with increasing density. Beyond just mapping PDs, we

Received: March 30, 2021

Revised: $\quad$ May 25, 2021

Published: June 4, 2021

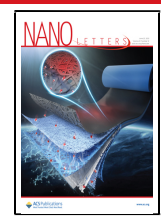




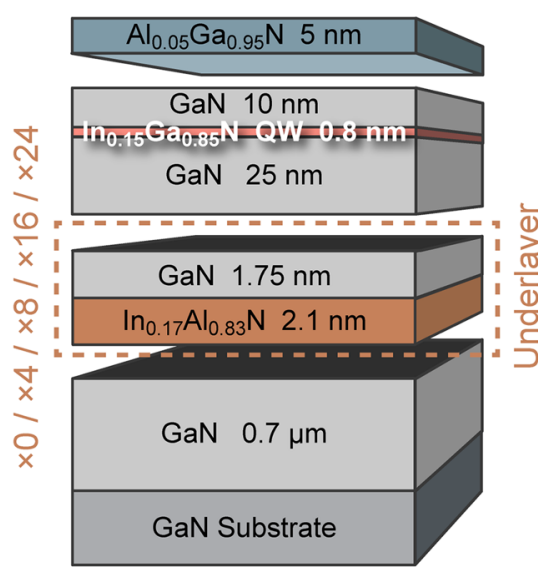

b

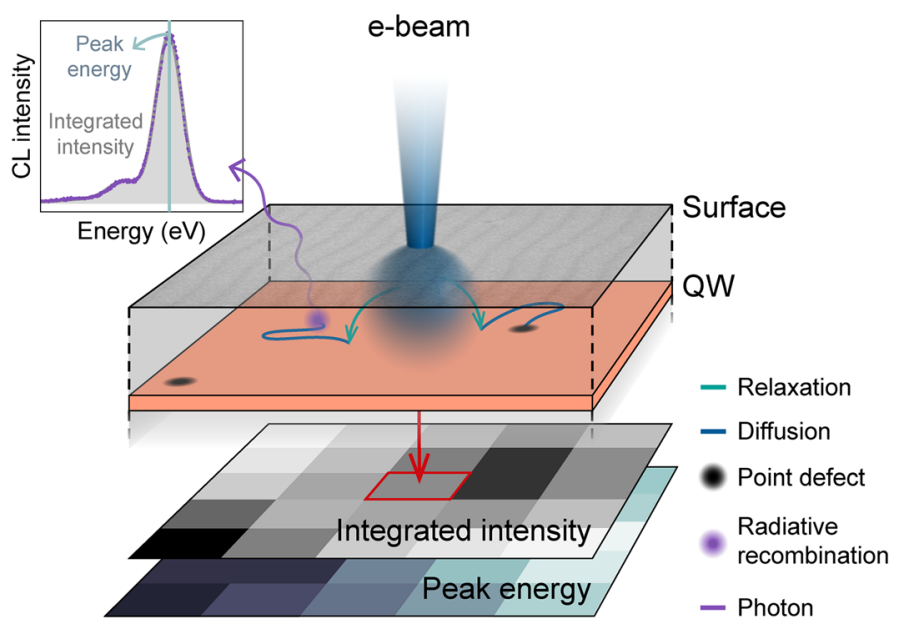

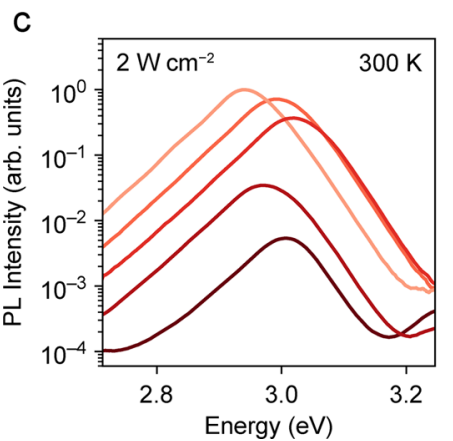

d
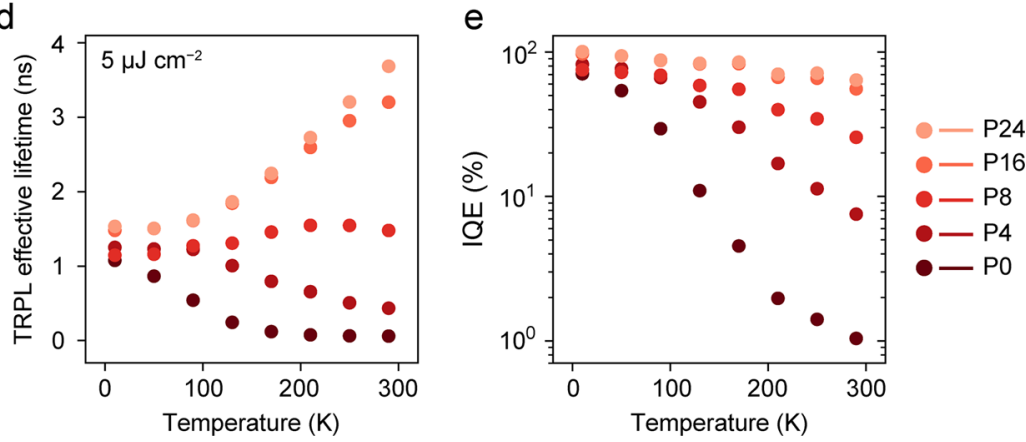

Figure 1. (a) Schematic of the sample design: five samples were prepared with $0,4,8,16$, and 24 periods in the SL; these samples are referred to as P0-P24, respectively. A thin QW allows for shorter diffusion lengths, and the top GaN layer is only $10 \mathrm{~nm}$ to excite close to the QW. (b) Diagram depicting CL imaging of a QW. Examples of possible carrier paths from one excitation position are illustrated with transfer to the QW followed by radiative recombination or recombination at a PD. Photons are globally collected to generate a CL spectrum for this position (example top left). This process is repeated as the beam is scanned over the area of interest, and all collected spectra are fitted to generate QW integrated intensity and peak energy images of this area. PDs present as dark areas in the intensity image since they locally reduce radiative recombination. (c) Roomtemperature PL spectra showing the QW emission of all samples at an excitation power density of $2 \mathrm{~W} \mathrm{~cm}{ }^{-2}$; peak intensities scale with the underlayer thickness. (d) The relation of effective carrier lifetime to temperature for each sample gained from TRPL at an excitation fluence of $5 \mu \mathrm{J}$ $\mathrm{cm}^{-2}$, along with (e) corresponding temperature-dependent IQE extracted from these effective lifetimes. Effective lifetimes and IQEs increase for samples with thicker underlayers, indicating a reduction in defect density.

also use CL images to explore their interaction with carrier dynamics in the well. We evidence the direct impact of PDs on carrier diffusion lengths and demonstrate how higher carrier densities strongly reduce the influence of PDs on the CL intensity.

We grew our samples by metal-organic vapor phase epitaxy, using $c$-plane freestanding $\mathrm{GaN}$ substrates (dislocation density $\sim 10^{6} \mathrm{~cm}^{-2}$ ) to minimize the influence of threading dislocations on our results. A lattice-matched $\operatorname{In}_{0.17} \mathrm{Al}_{0.83} \mathrm{~N} / \mathrm{GaN}$ superlattice (SL) was chosen as an underlayer to bury surface defects. $^{21,23}$ Five samples with varying underlayer thickness were grown by changing the number of SL periods, $n$; these samples are then labeled as $\mathrm{Pn}$ (Figure 1a). We grew a single three-monolayer $(\sim 0.8 \mathrm{~nm}) \mathrm{In}_{0.15} \mathrm{Ga}_{0.85} \mathrm{~N} / \mathrm{GaN}$ QW followed by a $10 \mathrm{~nm}$ top $\mathrm{GaN}$ barrier, before capping the sample with 5 $\mathrm{nm} \mathrm{Al}_{0.05} \mathrm{Ga}_{0.95} \mathrm{~N}$ to avoid surface recombination.

These samples are tailored for high-resolution CL. During $\mathrm{CL}$ acquisition, the electron beam raster-scans the sample surface to gather data at evenly spaced positions. At each position, the sample is locally excited while luminescence is globally collected, as depicted in Figure 1b. Electron-hole pairs generated within the electron-beam excitation volume rapidly relax to the QW (green arrows), diffusing laterally (blue paths) until they reach a $\mathrm{PD}$, or until they undergo radiative recombination. Photons emitted by the radiative recombinations (purple arrow) are collected to obtain a CL spectrum for each excitation position. By fitting the QW emission at every pixel in this full hyperspectral map (see Supporting Information (SI) Section 2), we generate both a QW integrated intensity image and a peak emission energy image of the scanned area. For most CL measurements in this work, the pixel spacing is $18 \mathrm{~nm}$ with a dwell time of $3 \mathrm{~ms}$.

Since photons are globally collected, the CL spectrum recorded for one position is spatially averaged over a QW area related to (i) the carrier diffusion length and (ii) the excitation volume. These parameters are often not controlled in QW CL studies, which can limit the spatial resolution to hundreds of nanometers or more. Here, we discuss how we minimize both parameters in our samples to obtain high-resolution images.

Carrier diffusion lengths can be reduced by lower diffusion coefficients and shorter carrier lifetimes. The diffusion coefficient in InGaN/GaN QWs is mainly controlled by temperature, starting from near zero at $10 \mathrm{~K}$ and increasing until it saturates at around $200 \mathrm{~K}^{24}$ Consequently, we reach 

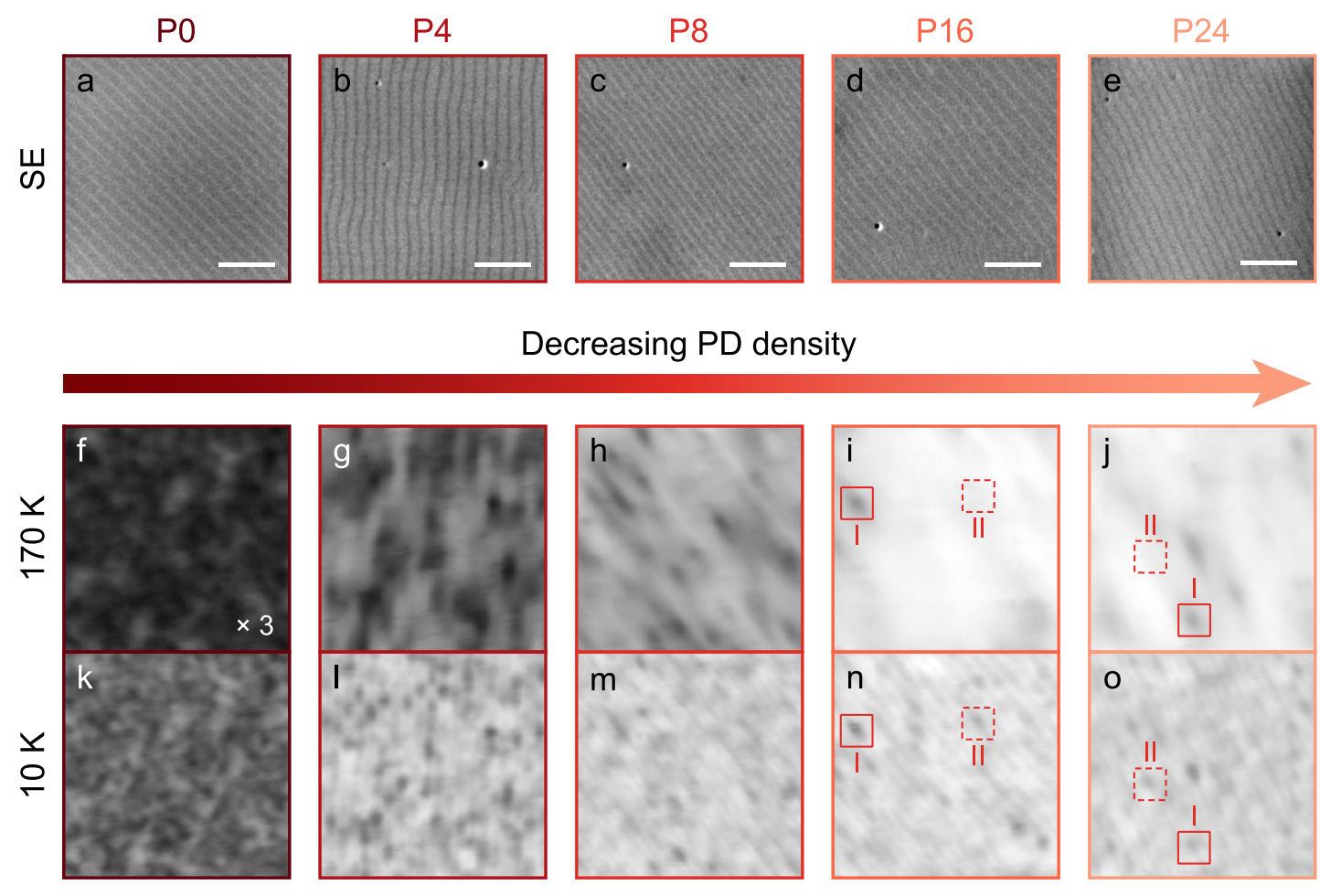

\section{Decreasing PD density}
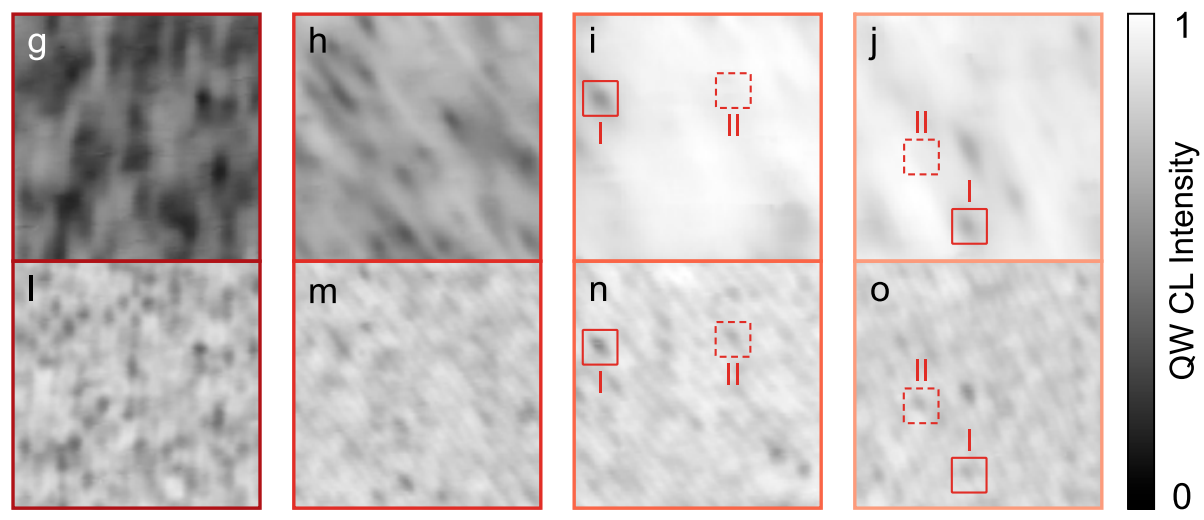

Figure 2. (a-e) SE images for each sample (P0 - P24); step-edges are clear as parallel lines, and V-shaped pits marking the position of threading dislocations are visible as dark spots. All are $2 \times 2 \mu \mathrm{m}^{2}$ with a $500 \mathrm{~nm}$ scale bar. Panels $(\mathrm{f}-\mathrm{j})$ and $(\mathrm{k}-\mathrm{o})$ show the QW CL integrated intensity images of the same $2 \times 2 \mu \mathrm{m}^{2}$ area for each sample at 170 and $10 \mathrm{~K}$, respectively. Dark regions are a direct consequence of nonradiative recombination at PDs and decrease in density as the SL periods are increased. Each image is plotted on an absolute intensity scale from 0-1 (normalized to the highest intensity point in P24). The red squares in panels (i), (j), (n), and (o) indicate examples of type-I PDs with a strong impact on high-temperature intensity (solid line) and type-II PDs with a lesser impact (dashed line). All images obtained at a probe current of 200 $\mathrm{pA}$. The $170 \mathrm{~K}$ intensity for $\mathrm{P} 0$ has been multiplied by three to make the image visible.

the highest resolution at cryogenic temperatures. Meanwhile, using an ultrathin QW (Figure 1a) significantly reduces the carrier radiative lifetime by maximizing electron-hole wave function overlap, ${ }^{25}$ further decreasing diffusion lengths.

To minimize the excitation volume, we use an electron-beam acceleration voltage of only $1.5 \mathrm{kV}$. At this voltage, $95 \%$ of the incident energy is absorbed within the top $20 \mathrm{~nm}$ of the sample (SI Section 3), so we chose a thin $10 \mathrm{~nm}$ top GaN layer to excite close to the QW (Figure 1a).

Before moving on to CL results, let us initially gauge the macroscale properties of our samples by conventional methods. The room-temperature photoluminescence (PL) spectra shown in Figure 1c clearly demonstrate the rise in QW efficiency achieved by the underlayer, with the peak intensity increasing by two orders of magnitude from P0 to P24 (for explanation of minor shifts in peak energy, see SI Section 7). This efficiency improvement is underscored by the striking contrast in QW effective lifetimes, gained from fitting time-resolved PL (TRPL) decay curves at early delays (Figure 1d) (see SI Section 4). The lifetime behavior of P24 indicates it is dominated by radiative recombination across the full temperature range, since it exactly matches radiative lifetime behavior in an ideal $\mathrm{QW} .^{26-29}$ For other samples, as the number of SL periods is reduced the high-temperature effective lifetimes decrease due to thermal activation of defect-assisted recombination. ${ }^{5,30}$ We quantify the IQE of all samples with a simple treatment comparing the initial TRPL intensity to the effective lifetime (SI Section 4). ${ }^{31}$ Results are displayed in Figure 1e: as expected, P24 maintains a high IQE of $64 \pm 3 \%$ at room temperature, which matches that measured for a similar sample in another study. ${ }^{23}$ Samples with fewer SL periods present much lower efficiencies, with the IQE of P0 falling to only $\sim 1 \%$ at room temperature. Overall, the results presented in Figure 1c-e confirm that the defect density in the InGaN/GaN single QW significantly reduces from P0 to P24. With only these measurements, however, it is difficult to quantify the defect density, let alone identify their nature.

To spatially resolve and directly analyze the PDs, we turn our attention to CL results (Figure 2). Atomic-height stepedges are noticeable in all $2 \times 2 \mu \mathrm{m}^{2}$ secondary-electron (SE) images (Figure $2 \mathrm{a}-\mathrm{e}$ ) as a series of parallel lines, indicating ideal step-flow growth (see SI Section 5 for complementary atomic force microscopy). Additionally, very few dislocationinduced V-pits ${ }^{32}$ are present due to the growth on freestanding GaN substrates.

We focus on CL integrated intensity images obtained by fitting the $\mathrm{QW}$ emission from hyperspectral data (Figure $2 \mathrm{f}-$ o). Two temperatures were chosen for CL characterization: 10 $\mathrm{K}$, at which the diffusivity in the well is near zero so resolution is only limited by the excitation volume, ${ }^{24}$ and $170 \mathrm{~K}$, which allows us to probe the high-temperature regime while retaining sufficient signal-to-noise ratio for the most defective samples. The images obtained at $10 \mathrm{~K}$ and $170 \mathrm{~K}$ for each sample are from precisely the same area displayed in the SE images. The electron-beam probe current was set to $200 \mathrm{pA}$, which results in a carrier density near the peak-IQE condition for highquality InGaN/GaN QWs (SI Section 3).

We observe the impact from defects as local regions of low intensity in the $170 \mathrm{~K}$ results (Figure $2 \mathrm{f}-\mathrm{j}$ ). Since dislocations identified in the SE images do not correlate with the dark 
regions, we attribute this low intensity to nonradiative recombination at PDs buried in the QW. The density of dark spots clearly decreases with increasing SL periods, directly indicating a decline in PD density in accordance with the PDreducing behavior of the underlayer. ${ }^{21}$ Thus, these CL images reveal the nanoscale origin of the increasing IQE from P0 to P24 in Figure 1e. The elongated shape of PD dark regions in most samples (Figure $2 \mathrm{~h}-\mathrm{j}$ ) is a result of asymmetric diffusion in the QWs as discussed later in our diffusion analysis.

Upon cooling to $10 \mathrm{~K}$ (Figure $2 \mathrm{k}-\mathrm{o}$ ), the impact from PDs is noticeably suppressed, as expected since the reduction in diffusion length means fewer carriers can reach PDs. Nonradiative recombination at defects also usually requires multiple-phonon emission, which is less likely at low temperatures. ${ }^{5}$ Nevertheless, we still see some influence of PDs on the QW intensity. Observing effects from defects at cryogenic temperatures is not surprising; even at $10 \mathrm{~K}$ the IQEs of the more defective samples are only $~ 70 \%$ rather than $100 \%$ (Figure 1e). Such nonradiative recombination at low temperatures has previously been explained through tunnelling-assisted transitions to defect levels. ${ }^{30}$ With the improved spatial resolution of under $90 \mathrm{~nm}$ at this temperature, we can be confident we are observing individual PDs as long as densities remain below $10^{17} \mathrm{~cm}^{-3}$. This resolution estimate includes the effects from both the excitation volume and carrier diffusion (SI Section 3).

Comparing the $10 \mathrm{~K}$ and $170 \mathrm{~K}$ results for P16 and P24 (Figure 2i,j,n,o), we can distinguish two different types of PD: one type leads to greater local intensity reduction at high temperature (type-I), whereas the other has much less effect (type-II). With a clear decrease in the number of dark regions at $170 \mathrm{~K}$ with increasing SL periods, we can already suggest the type-I defect density is reduced by the underlayer. However, quantifying the density of each type of defect requires a more detailed analysis of the CL images.

To estimate PD densities, we need to differentiate the two kinds of PDs using their contrasting high-temperature behavior as a criterion. This task is made more challenging by the loss of spatial resolution at $170 \mathrm{~K}$ due to increased carrier diffusion. Nonetheless, we can use $10 \mathrm{~K}$ images to accurately determine the position of PDs since diffusion length is near zero at this temperature. Therefore, we established a general two-step procedure: (i) use the Laplacian of Gaussian method ${ }^{33}$ to automatically detect dark regions in a $10 \mathrm{~K}$ image, then (ii) superimpose the positions of these regions on a $170 \mathrm{~K} \mathrm{CL}$ image of the same sample area. If a position is associated with an intensity below a defined limit at $170 \mathrm{~K}, I_{\text {lim }}$, it is identified as a type-I defect due to their strong impact at high temperature.

An example of the procedure applied to P4 is shown in Figure 3a,b. We note that PDs of the same type can lead to different levels of local intensity reduction; these slight variations could arise from the random indium atomic configurations around each PD or from the specific vertical position of each PD within the QW as related to the electronhole wave function overlap. ${ }^{34}$ Although this variation means our procedure may not correctly detect and identify every PD, the design ensures reproducibility and avoids bias arising from counting/differentiating PDs manually. For more information on the PD counting method, see SI Section 6. Once we have the $2 \mathrm{D}$ defect densities from this method, we calculate the 3D densities using the full width at half-maximum of the QW indium profile measured by scanning transmission electron
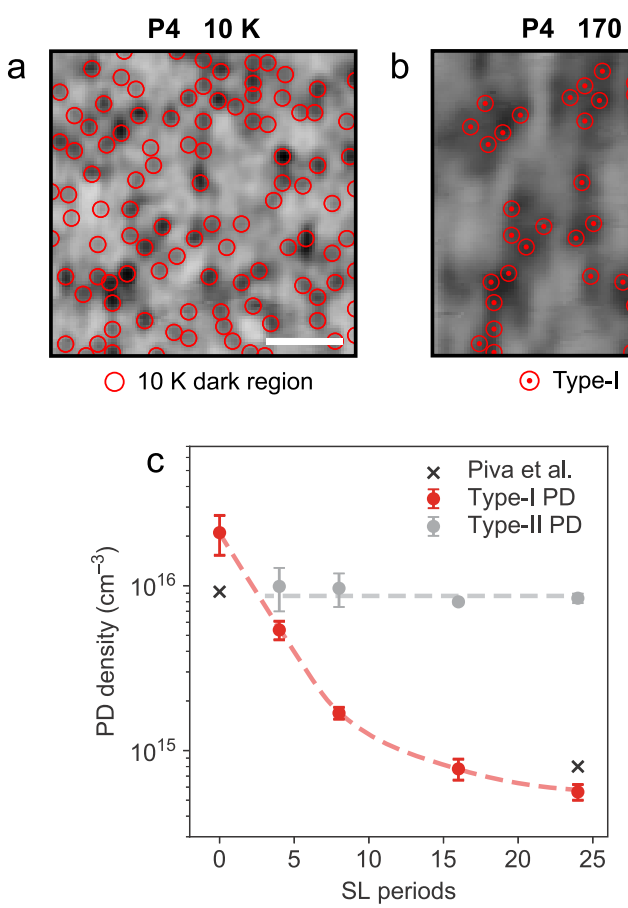

Figure 3. (a) P4 $10 \mathrm{~K} \mathrm{CL}$ intensity image from Figure 2 with red circles enclosing dark regions detected by the Laplacian of Gaussian method which could be type-I or type-II PDs (intensity scale: $0.4-1$ ). The detected dark regions which lead to an intensity at $170 \mathrm{~K}$ below a set limit, $I_{\text {lim }}$ are identified as type-I PDs as shown in (b) (intensity scale: $0-1, I_{\text {lim }}=0.32$ ). Scale bars are $500 \mathrm{~nm}$. (c) Type-I and type-II PD densities in the QW for each sample, demonstrating the drastic decrease in type-I PD density with increasing underlayer thickness. Densities of midgap PDs calculated by Piva et al. ${ }^{35}$ on very similar samples to P0 and P24 are also displayed. Dashed lines are guides to the eye.

microscopy (SI Section 7) since the PDs are contained within the well.

The measured PD densities are shown in Figure 3c. We note that for P0 the very high concentration of type-I PDs overwhelmed any effect from type-II PDs, preventing the estimation of the type-II defect density. With a decrease in the concentration of type-I defects in the QW from $10^{16} \mathrm{~cm}^{-3}$ to only $10^{14} \mathrm{~cm}^{-3}$ with increasing SL periods, we propose that type-I PDs are the key defects incorporated into the underlayer. In contrast, the concentration of type-II PDs is rather insensitive to the underlayer thickness. We conclude that the drastic increase in room-temperature IQE from P0 to P24 (Figure 1e) is entirely due to the reduction in type-I PD density, while type-II defects have only a minor effect. The strong impact of type-I PDs on CL intensity and IQE indicates they possess energy levels near the middle of the InGaN bandgap, since such midgap states are known to be efficient nonradiative recombination centers. ${ }^{2,36}$

Our CL-derived type-I PD densities are in good agreement with previous results from macroscale measurements by Piva et al. $^{35}$ (Figure 3c), which corroborates that we are spatially resolving individual PDs. Furthermore, their results also associate these defects with a near-midgap energy level and identify another energy level near the valence band edge which could match our type-II defects. Other macroscale studies have also detected two types of PDs in InGaN/GaN QWs. ${ }^{17,19}$ Evidence suggests midgap (type-I) defects are intrinsic 

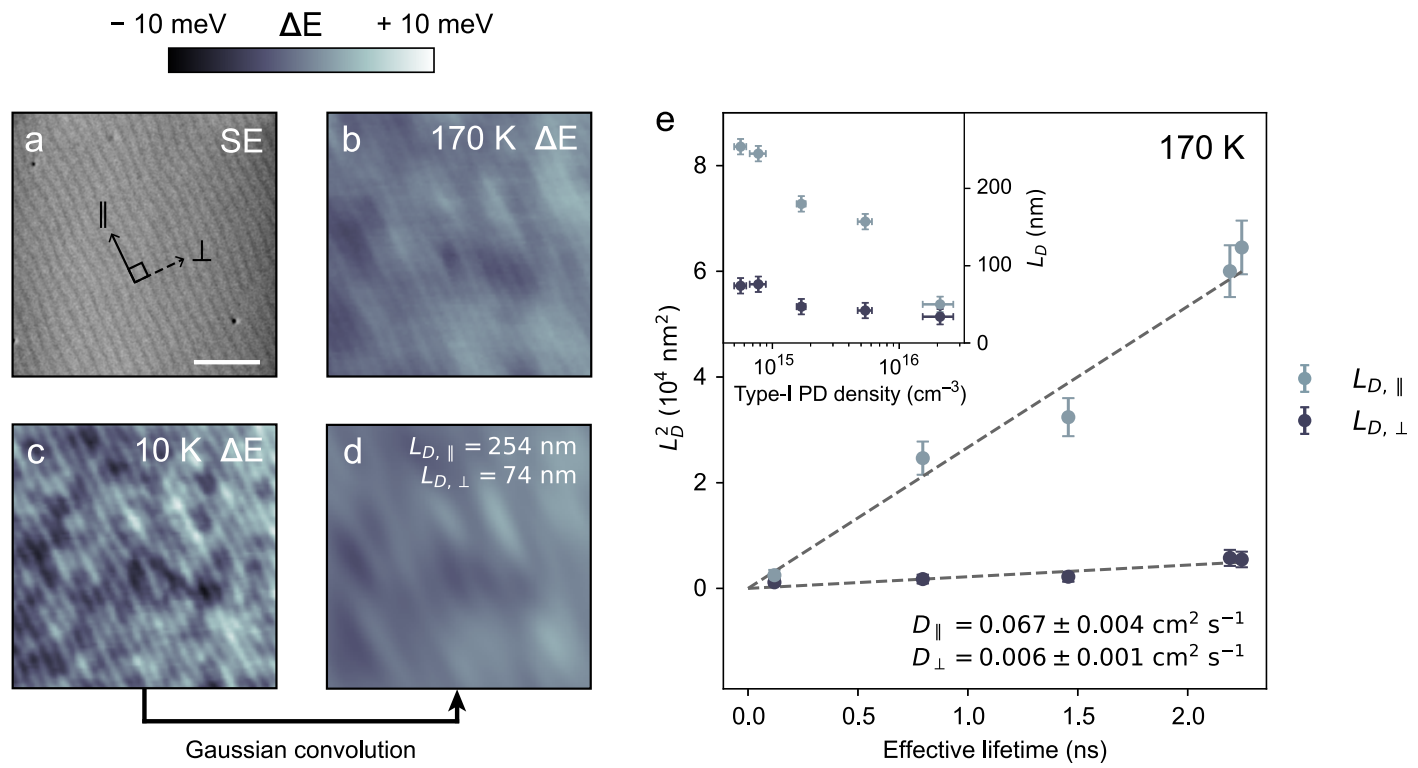

Figure 4. (a) SE image for P24 as in Figure 2e with directions parallel (\|) and perpendicular $(\perp)$ to the step-edges highlighted (scale bar $500 \mathrm{~nm}$ ). $(b, c)$ Images of QW peak energy from the same area as in (a) at $170 \mathrm{~K}$ and $10 \mathrm{~K}$, respectively. Energy modulation periodic with the step-edges is clear, and this modulation restricts carrier diffusion perpendicular to the step-edges. Restricted diffusion is confirmed by (d) the image calculated by Gaussian convolution fitting of the $10 \mathrm{~K}$ data in (c) to the $170 \mathrm{~K}$ data in (b), since the extracted perpendicular diffusion length $\left(L_{\mathrm{D}, \perp}\right)$ is much shorter than the parallel length $\left(L_{\mathrm{D}, \|}\right)$. (e) Both diffusion lengths squared plotted against the effective lifetime of each sample. Linear fits are used to calculate the diffusivities at $170 \mathrm{~K}, D_{\|}$and $D_{\perp}$; the large difference in these diffusion coefficients emphasizes the anisotropic diffusion in our QWs. The reduced $\chi$-squared for these fits were 1.5 and 0.9 for parallel and perpendicular diffusion length data, respectively. The inset shows the severe shortening of diffusion lengths at $170 \mathrm{~K}$ with increasing type-I PD density.

nitrogen-vacancy complexes, ${ }^{21,22,37}$ whereas the type-II defect may be linked to impurities such as oxygen or carbon. ${ }^{38}$

Having characterized these nonradiative PDs, we now investigate how their density impacts the carrier diffusion length in the well, which is a critical parameter for the performance of optoelectronic devices such as micro-LEDs. CL has been used to gauge carrier diffusion through various methods, often requiring a specific metallic mask, reference samples, or precise knowledge of the excitation volume. ${ }^{39-42}$ Here, we estimate diffusion lengths without having to take any new measurements but instead from a direct analysis of the hyperspectral maps we already used to generate the images in Figure 2.

At $10 \mathrm{~K}$, carriers in InGaN/GaN QWs have near-zero diffusivity, and therefore negligible diffusion lengths. As the temperature is raised, the diffusion constant increases before saturating at $\sim 200 \mathrm{~K}^{24}$ The resulting increase in diffusion length reduces the spatial resolution at higher temperatures, as shown in Figure 2 comparing the $170 \mathrm{~K}$ images to those at 10 K.

Neglecting drift effects, carriers injected into the well diffuse from each point into 2D Gaussian distributions with a standard deviation, $\sigma_{\mathrm{d}}$, linked to the diffusion length, $L_{\mathrm{D}}$, by $L_{\mathrm{D}}=\sigma_{\mathrm{d}} \sqrt{2}{ }^{43}$ We can therefore straightforwardly model the effect of diffusion on our CL images by convoluting the negligibly diffused $10 \mathrm{~K}$ images with $2 \mathrm{D}$ Gaussians. Using the convolution Gaussian's $\sigma_{\mathrm{d}}$ as a fitting parameter, we can fit each $10 \mathrm{~K}$ image to the $170 \mathrm{~K}$ image of the same area, extracting $L_{\mathrm{D}}$ at $170 \mathrm{~K}$ from the optimum fit for each sample. This method analyses average $2 \mathrm{D}$ diffusion across the entire image, rather than inspecting only one local feature.

Using the CL intensity for this procedure is not ideal, as we cannot account for the thermal activation of nonradiative recombination at PDs. Instead, we use the QW peak energy images of the same areas as in Figure 2, which are directly "smoothened" as the diffusion length increases (see SI Section 8 for further detail). The importance of using CL energy rather than intensity for diffusion analysis has been emphasized for the case of threading dislocations in $\mathrm{GaN}^{44}$ Since we are not interested in macroscopic changes in the peak energy with temperature, we subtract the mean peak energy from each image to map deviation from average peak energy, $\Delta E$. These images then reflect inhomogeneity across the QW.

An example of this diffusion analysis applied to P24 is shown in Figure $4 a-d$ (see SI Section 8 for analysis of all samples). Inspecting Figure $4 c$, we observe modulation in the $\mathrm{QW}$ emission energy by $\sim 10 \mathrm{meV}$ with the same periodicity as the step-edges in the SE image (Figure 4a). While this modulation likely originates from the impact of step-edges on the bottom interface of the well, ${ }^{45}$ it is beyond the scope of this study. However, the modulation has a direct influence on the carrier dynamics within the $\mathrm{QW}$ by presenting an energy barrier to diffusion perpendicular to the step-edges. This leads to anisotropic carrier diffusion in the QW, which we account for by adjusting the convolution Gaussian to contain two different perpendicular standard deviations. One standard deviation was fixed parallel to the step-edge direction, resulting in a diffusion length $L_{\mathrm{D}, \|}$, whereas the other was set perpendicular to the step-edges, giving $L_{\mathrm{D}, \perp}$. The fitting result from convolution of the $10 \mathrm{~K}$ energy map is shown in Figure $4 \mathrm{~d}$ and compares well with the real $170 \mathrm{~K}$ data (Figure $4 \mathrm{~b}$ ). The computed values for the diffusion lengths confirm the anisotropic diffusion, with $L_{\mathrm{D}, \|}$ being over three times larger than $L_{\mathrm{D}, \perp}$. This step-edge induced anisotropy is likely stronger in our QWs since the effect of interface roughness on the carrier confinement energy is magnified for thinner wells.

$L_{\mathrm{D}, \|}$ and $L_{\mathrm{D}, \perp}$ for all samples are plotted against their type-I $\mathrm{PD}$ densities in the inset of Figure $4 \mathrm{e}$, with every sample 


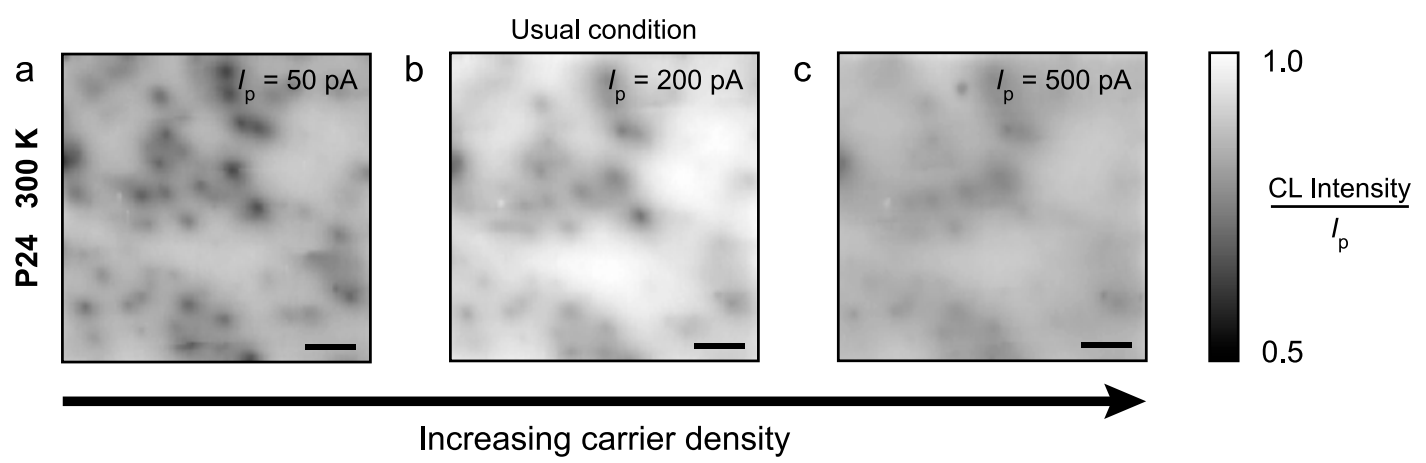

Figure 5. Large-scale QW integrated intensity images for P24 at $300 \mathrm{~K}$ with the probe current $\left(I_{\mathrm{p}}\right)$ varied from (a) $50 \mathrm{pA}$ (carrier density in the high $10^{11} \mathrm{~cm}^{-2}$ range) to (b) $200 \mathrm{pA}$ (condition used for all other results, $10^{12} \mathrm{~cm}^{-2}$ range) to (c) $500 \mathrm{pA}$ (low $10^{13} \mathrm{~cm}^{-2}$ range). PDs become saturated as the probe current, and hence the carrier density, is increased. All results are plotted on an intensity scale of $0.5-1$, normalized relative to probe current and maximum intensity at $200 \mathrm{pA}$. Scale bars are $1 \mu \mathrm{m}$.

exhibiting anisotropic diffusion. Crucially, both $L_{\mathrm{D}, \|}$ and $L_{\mathrm{D}, \perp}$ are severely limited by large PD concentrations, with $L_{D, \|}$ decreasing by over $80 \%$ from P24 to P0. In other words, despite the increased diffusion coefficient at raised temperatures, carriers in the more defective samples are unable to diffuse far since they quickly undergo nonradiative recombination at PDs. We link this trend to the effective lifetimes gained from TRPL (Figure $1 \mathrm{~d}$ ), $\tau_{\text {eff }}$, through $L_{\mathrm{D}}=2 \sqrt{D \tau_{\text {eff }}}$ where $D$ is the diffusivity at $170 \mathrm{~K}$. The excellent fits to the parallel/perpendicular data obtained using this relation (Figure 4 e) underscore the validity of our diffusion analysis. $D_{\|}$, which represents unrestricted diffusion in the well, is in reasonable agreement with diffusion coefficients previously found for InGaN/GaN QWs. ${ }^{24}$ Overall, we have directly demonstrated that $\mathrm{PD}$ density is a critical parameter controlling diffusion in QWs, re-emphasizing that high-IQE samples are required to study intrinsic carrier dynamics.

While PD density influences the carrier dynamics in the well, the carrier density likewise influences PDs. Specifically, macroscale measurements indicate that the carrier density strongly impacts defect-related nonradiative recombination. ${ }^{46}$ In continuous-excitation CL, the local carrier density increases with the generation rate and lifetime of the carriers. Since the generation rate is proportional to the CL electron-beam probe current, $I_{\mathrm{p}}{ }^{47}$ this current directly affects the carrier density and hence defect-related recombination in the $\mathrm{QW}$, yet $I_{\mathrm{p}}$ is not commonly varied in CL defect studies.

In Figure 5, we investigate how carrier density alters the nanoscale nonradiative behavior of PDs by mapping the QW intensity on the same area of P24 at three different probe currents. Because of the low density of type-I defects in P24, the images can be taken at a higher temperature and larger scale than previous results while still being able to resolve PDs. The images are normalized relative to $I_{\mathrm{p}}$ to give an indication of how IQE is affected. On increasing $I_{\mathrm{p}}$, the impact from PDs is greatly reduced since defect-assisted nonradiative recombination usually dominates at lower carrier densities. ${ }^{46}$ As the carrier density is increased, radiative recombination and then Auger recombination progressively play larger roles. Both of these processes are unlikely to be strongly spatially correlated with defects, and hence they suppress any influence of PDs on the CL intensity as in Figure 5c. We note that the brightness of the $I_{\mathrm{p}}=200 \mathrm{pA}$ image (Figure $5 \mathrm{~b}$ ) suggests this condition is close to peak-IQE, which is in agreement with the calculated CL carrier density of $\sim 10^{12} \mathrm{~cm}^{-2}$ (see SI Section 3 ). As well as demonstrating the nanoscale dependence of PD nonradiative recombination on the carrier density, these results emphasize that probe current, though often overlooked, must be carefully selected for optimal defect imaging in CL.

In summary, combining $\mathrm{CL}$ at an acceleration voltage of only $1.5 \mathrm{kV}$ with specific sample design, we spatially resolved and analyzed individual nonradiative PDs buried in InGaN/ GaN single QWs. Using just two hyperspectral maps of each sample at different temperatures, we extracted information on PD types, PD densities, and carrier diffusion lengths, all through direct observation and straightforward analysis methods. We identified two different types of PDs: nearmidgap defects with a strong impact on CL intensity and IQE which can be incorporated into an indium-containing underlayer (type-I), and defects with a lesser impact on efficiency which are not affected by the underlayer (type-II). The density of type-I PDs was estimated to range from $10^{14} \mathrm{~cm}^{-3}$ up to $10^{16} \mathrm{~cm}^{-3}$ with decreasing underlayer thickness, highlighting the capability of our methodology to image PDs even up to high densities. Furthermore, we measured diffusion lengths from CL peak energy images, demonstrating their dependence on the PD concentration, and showed that carrier density significantly affects the influence of PDs on CL intensity. Overall, this comprehensive study serves as a proof-of-concept for nanoscale imaging and direct analysis of nonradiative PDs in QWs and lays the foundation for advanced future studies such as applying time-resolved CL to a single buried PD.

\section{ASSOCIATED CONTENT}

\section{Supporting Information}

The Supporting Information is available free of charge at https://pubs.acs.org/doi/10.1021/acs.nanolett.1c01295.

Further details on (1) experimental methods; (2) CL hyperspectral fitting; (3) carrier injection in CL; (4) TRPL; (5) atomic force microscopy; (6) point defect counting; (7) transmission electron microscopy and transition energy simulations; and (8) complete diffusion analysis (PDF)

\section{AUTHOR INFORMATION}

\section{Corresponding Author}

Thomas F. K. Weatherley - Institute of Physics, Ecole Polytechnique Fédérale de Lausanne (EPFL), CH-1015 Lausanne, Switzerland; 이이.org/0000-0002-77372852; Email: thomas.weatherley@epfl.ch 


\section{Authors}

Wei Liu - Institute of Physics, Ecole Polytechnique Fédérale de Lausanne (EPFL), CH-1015 Lausanne, Switzerland

Vitaly Osokin - Department of Physics, The Clarendon Laboratory, University of Oxford, Oxford OX1 3PU, United Kingdom

Duncan T. L. Alexander - Institute of Physics, Ecole Polytechnique Fédérale de Lausanne (EPFL), CH-1015 Lausanne, Switzerland; 이이.org/0000-0003-4350-8587

Robert A. Taylor - Department of Physics, The Clarendon Laboratory, University of Oxford, Oxford OX1 3PU, United Kingdom; (ㅇ) orcid.org/0000-0003-2578-9645

Jean-François Carlin - Institute of Physics, Ecole Polytechnique Fédérale de Lausanne (EPFL), CH-1015 Lausanne, Switzerland

Raphaël Butté - Institute of Physics, Ecole Polytechnique Fédérale de Lausanne (EPFL), CH-1015 Lausanne, Switzerland

Nicolas Grandjean - Institute of Physics, Ecole Polytechnique Fédérale de Lausanne (EPFL), CH-1015 Lausanne, Switzerland

Complete contact information is available at:

https://pubs.acs.org/10.1021/acs.nanolett.1c01295

\section{Notes}

The authors declare no competing financial interest.

\section{ACKNOWLEDGMENTS}

This work was supported by the Swiss National Science Foundation through Grant 200021E 175652. The CIME at EPFL is acknowledged for access to its facilities, and Dr. B. Bártová at CIME is thanked for the focused ion beam preparation. We thank N. Tappy (EPFL), Dr. C. Haller (EPFL), and Prof. G. Callsen (Uni Bremen), for useful discussion.

\section{REFERENCES}

(1) Bourgoin, J.; Lannoo, M. Point Defects in Semiconductors II: Experimental Aspects; Springer-Verlag: Heidelberg, 1983.

(2) Shockley, W.; Read, W. T. Statistics of the recombinations of holes and electrons. Phys. Rev. 1952, 87, 835-842.

(3) Aharonovich, I.; Englund, D.; Toth, M. Solid-state single-photon emitters. Nat. Photonics 2016, 10, 631-641.

(4) Bradac, C.; Gao, W.; Forneris, J.; Trusheim, M. E.; Aharonovich, I. Quantum nanophotonics with group IV defects in diamond. Nat. Commun. 2019, 10, 1-13.

(5) Henry, C. H.; Lang, D. V. Nonradiative capture and recombination by multiphonon emission in GaAs and GaP. Phys. Rev. B 1977, 15, 989-1016.

(6) Kressel, H. The effect of crystal defects on optoelectronic devices. Semiconductors and Semimetals; Academic Press: London, 1981; Vol. 16, Chapter 1.

(7) Ball, J. M.; Petrozza, A. Defects in perovskite-halides and their effects in solar cells. Nature Energy 2016, 1, 16149.

(8) Park, J. S.; Kim, S.; Xie, Z.; Walsh, A. Point defect engineering in thin-film solar cells. Nature Reviews Materials 2018, 3, 194-210.

(9) Bourrellier, R.; Meuret, S.; Tararan, A.; Stéphan, O.; Kociak, M.; Tizei, L. H.; Zobelli, A. Bright UV single photon emission at point defects in h-BN. Nano Lett. 2016, 16, 4317-4321.

(10) Sang, X.; Xie, Y.; Lin, M. W.; Alhabeb, M.; Van Aken, K. L.; Gogotsi, Y.; Kent, P. R.; Xiao, K.; Unocic, R. R. Atomic defects in monolayer titanium carbide (Ti3C2Tx) MXene. ACS Nano 2016, 10, 9193-9200.

(11) Jiang, Y.; Chen, Z.; Han, Y.; Deb, P.; Gao, H.; Xie, S.; Purohit, P.; Tate, M. W.; Park, J.; Gruner, S. M.; Elser, V.; Muller, D. A.
Electron ptychography of $2 \mathrm{D}$ materials to deep sub-ångström resolution. Nature 2018, 559, 343-349.

(12) Feng, J.; Deschout, H.; Caneva, S.; Hofmann, S.; Lončarić, I.; Lazić, P.; Radenovic, A. Imaging of Optically Active Defects with Nanometer Resolution. Nano Lett. 2018, 18, 1739-1744.

(13) Edelberg, D.; et al. Approaching the Intrinsic Limit in Transition Metal Diselenides via Point Defect Control. Nano Lett. 2019, 19, 4371-4379.

(14) Ziatdinov, M.; Dyck, O.; Li, X.; Sumpter, B. G.; Jesse, S.; Vasudevan, R. K.; Kalinin, S. V. Building and exploring libraries of atomic defects in graphene: Scanning transmission electron and scanning tunneling microscopy study. Science Advances 2019, 5, No. eaaw8989.

(15) Jagsch, S. T.; Triviño, N. V.; Lohof, F.; Callsen, G.; Kalinowski, S.; Rousseau, I. M.; Barzel, R.; Carlin, J.-F.; Jahnke, F.; Butté, R.; Gies, C.; Hoffmann, A.; Grandjean, N.; Reitzenstein, S. A quantum optical study of thresholdless lasing features in high- $\beta$ nitride nanobeam cavities. Nat. Commun. 2018, 9, 564.

(16) Rittweger, E.; Han, K. Y.; Irvine, S. E.; Eggeling, C.; Hell, S. W. STED microscopy reveals crystal colour centres with nanometric resolution. Nat. Photonics 2009, 3, 144-147.

(17) Armstrong, A.; Henry, T. A.; Koleske, D. D.; Crawford, M. H.; Lee, S. R. Quantitative and depth-resolved deep level defect distributions in InGaN/GaN light emitting diodes. Opt. Express 2012, 20, A812-A821.

(18) Armstrong, A. M.; Crawford, M. H.; Koleske, D. D. Contribution of deep-level defects to decreasing radiative efficiency of InGaN/GaN quantum wells with increasing emission wavelength. Appl. Phys. Express 2014, 7, 032101.

(19) Armstrong, A. M.; Bryant, B. N.; Crawford, M. H.; Koleske, D. D.; Lee, S. R.; Wierer, J. J. Defect-reduction mechanism for improving radiative efficiency in InGaN/GaN light-emitting diodes using InGaN underlayers. J. Appl. Phys. 2015, 117, 134501.

(20) Haller, C.; Carlin, J.-F.; Jacopin, G.; Martin, D.; Butté, R.; Grandjean, N. Burying non-radiative defects in InGaN underlayer to increase InGaN/GaN quantum well efficiency. Appl. Phys. Lett. 2017, $111,262101$.

(21) Haller, C.; Carlin, J.-F.; Jacopin, G.; Liu, W.; Martin, D.; Butté, R.; Grandjean, N. GaN surface as the source of non-radiative defects in InGaN/GaN quantum wells. Appl. Phys. Lett. 2018, 113, 111106.

(22) Chen, Y.; Haller, C.; Liu, W.; Karpov, S. Y.; Carlin, J.-F.; Grandjean, N. GaN buffer growth temperature and efficiency of InGaN/GaN quantum wells: The critical role of nitrogen vacancies at the GaN surface. Appl. Phys. Lett. 2021, 118, 111102.

(23) Haller, C.; Carlin, J.-F.; Mosca, M.; Rossell, M. D.; Erni, R.; Grandjean, N. InAlN underlayer for near ultraviolet InGaN based light emitting diodes. Appl. Phys. Express 2019, 12, 034002.

(24) Solowan, H. M.; Danhof, J.; Schwarz, U. T. Direct observation of charge carrier diffusion and localization in an InGaN multi quantum well. Jpn. J. Appl. Phys. 2013, 52, 08JK07.

(25) Berkowicz, E.; Gershoni, D.; Bahir, G.; Lakin, E.; Shilo, D.; Zolotoyabko, E.; Abare, A. C.; Denbaars, S. P.; Coldren, L. A. Measured and calculated radiative lifetime and optical absorption of $\mathrm{In}_{x} \mathrm{Ga}_{1-x} \mathrm{~N} / \mathrm{GaN}$ quantum structures. Phys. Rev. B 2000, 61, 1099411008 .

(26) Matsusue, T.; Sakaki, H. Radiative recombination coefficient of free carriers in GaAs-AlGaAs quantum wells and its dependence on temperature. Appl. Phys. Lett. 1987, 50, 1429-1431.

(27) Feldmann, J.; Peter, G.; Göbel, E. O.; Dawson, P.; Moore, K.; Foxon, C.; Elliott, R. J. Linewidth dependence of radiative exciton lifetimes in quantum wells. Phys. Rev. Lett. 1987, 59, 2337-2340.

(28) Andreani, L. C.; Tassone, F.; Bassani, F. Radiative lifetime of free excitons in quantum wells. Solid State Commun. 1991, 77, 641645.

(29) Hangleiter, A.; Langer, T.; Henning, P.; Ketzer, F. A.; Horenburg, P.; Korn, E. R.; Bremers, H.; Rossow, U. Radiative recombination in polar, non-polar, and semi-polar III-nitride quantum wells. Proc. SPIE 2017, 10104, 101040Q. 
(30) Hangleiter, A.; Langer, T.; Henning, P.; Ketzer, F. A.; Bremers, $\mathrm{H}$.; Rossow, U. Internal quantum efficiency of nitride light emitters: a critical perspective. Proc. SPIE 2018, 10532, 105321P.

(31) Langer, T.; Chernikov, A.; Kalincev, D.; Gerhard, M.; Bremers, H.; Rossow, U.; Koch, M.; Hangleiter, A. Room temperature excitonic recombination in GaInN/GaN quantum wells. Appl. Phys. Lett. 2013, 103, 202106.

(32) Chen, Y.; Takeuchi, T.; Amano, H.; Akasaki, I.; Yamada, N.; Kaneko, Y.; Wang, S. Y. Pit formation in GaInN quantum wells. Appl. Phys. Lett. 1998, 72, 710-712.

(33) Xiong, X.; Choi, B.-J. Comparative analysis of detection algorithms for corner and blob features in image processing. International Journal of Fuzzy Logic and Intelligent Systems 2013, 13, 284-290.

(34) David, A.; Hurni, C. A.; Young, N. G.; Craven, M. D. Fieldassisted Shockley-Read-Hall recombinations in III-nitride quantum wells. Appl. Phys. Lett. 2017, 111, 233501.

(35) Piva, F.; De Santi, C.; Caria, A.; Haller, C.; Carlin, J.-F.; Mosca, M.; Meneghesso, G.; Zanoni, E.; Grandjean, N.; Meneghini, M. Defect incorporation in In-containing layers and quantum wells: experimental analysis via deep level profiling and optical spectroscopy. J. Phys. D: Appl. Phys. 2021, 54, 025108.

(36) Dreyer, C. E.; Alkauskas, A.; Lyons, J. L.; Speck, J. S.; Van De Walle, C. G. Gallium vacancy complexes as a cause of Shockley-ReadHall recombination in III-nitride light emitters. Appl. Phys. Lett. 2016, 108, 141101.

(37) Han, D.-P.; Fujiki, R.; Takahashi, R.; Ueshima, Y.; Ueda, S.; Lu, W.; Iwaya, M.; Takeuchi, T.; Kamiyama, S.; Akasaki, I. n-type GaN surface etched green light-emitting diode to reduce non-radiative recombination centers. Appl. Phys. Lett. 2021, 118, 021102.

(38) Reshchikov, M. A.; Demchenko, D. O.; Usikov, A.; Helava, H.; Makarov, Y. Carbon defects as sources of the green and yellow luminescence bands in undoped GaN. Phys. Rev. B 2014, 90, 235203.

(39) Zarem, H. A.; Sercel, P. C.; Lebens, J. A.; Eng, L. E.; Yariv, A.; Vahala, K. J. Direct determination of the ambipolar diffusion length in GaAs/AlGaAs heterostructures by cathodoluminescence. Appl. Phys. Lett. 1989, 55, 1647-1649.

(40) Jahn, U.; Dhar, S.; Hey, R.; Brandt, O.; Miguel-Sánchez, J.; Guzmán, A. Influence of localization on the carrier diffusion in GaAs/ $(\mathrm{Al}, \mathrm{Ga}) \mathrm{As}$ and $(\mathrm{In}, \mathrm{Ga})(\mathrm{As}, \mathrm{N}) / \mathrm{GaAs}$ quantum wells: A comparative study. Phys. Rev. B 2006, 73, 125303.

(41) Pauc, N.; Phillips, M. R.; Aimez, V.; Drouin, D. Carrier recombination near threading dislocations in GaN epilayers by low voltage cathodoluminescence. Appl. Phys. Lett. 2006, 89, 161905.

(42) Gustafsson, A.; Bolinsson, J.; Sköld, N.; Samuelson, L. Determination of diffusion lengths in nanowires using cathodoluminescence. Appl. Phys. Lett. 2010, 97, 072114.

(43) Crank, J. The Mathematics of Diffusion, 2nd ed.; Oxford University Press: London, 1975; Chapter 3.

(44) Lähnemann, J.; Kaganer, V. M.; Sabelfeld, K. K.; Kireeva, A. E.; Jahn, U.; Chèze, C.; Calarco, R.; Brandt, O. Carrier diffusion in GaN - a cathodoluminescence study. III: Nature of nonradiative recombination at threading dislocations. 2020, 2009.14634. arXiv. http://arxiv.org/abs/2009.14634 (accessed May 17, 2021).

(45) Tanaka, M.; Sakaki, H. Atomistic models of interface structures

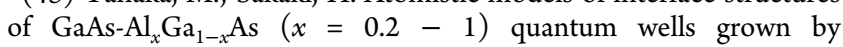
interrupted and uninterrupted MBE. J. Cryst. Growth 1987, 81, 153158.

(46) David, A.; Young, N. G.; Lund, C.; Craven, M. D. Review - The physics of recombinations in III-nitride emitters. ECS Journal of Solid State Science and Technology 2020, 9, 016021.

(47) Guthrey, H.; Moseley, J. A Review and Perspective on Cathodoluminescence Analysis of Halide Perovskites. Adv. Energy Mater. 2020, 10, 1903840. 